\title{
Bacterial communities in termite fungus combs are comprised of consistent gut deposits and contributions from the environment
}

\author{
Saria Otani ${ }^{1}$ (D) - Lars H. Hansen ${ }^{2,3} \cdot$ Søren J. Sørensen ${ }^{2} \cdot$ Michael Poulsen ${ }^{1}$
}

Received: 8 July 2014 / Accepted: 15 October 2015 /Published online: 30 October 2015

(C) The Author(s) 2015. This article is published with open access at Springerlink.com

\begin{abstract}
Fungus-growing termites (subfamily Macrotermitinae) mix plant forage with asexual spores of their plant-degrading fungal symbiont Termitomyces in their guts and deposit this blend in fungus comb structures, within which the plant matter is degraded. As Termitomyces grows, it produces nodules with asexual spores, which the termites feed on. Since all comb material passes through termite guts, it is inevitable that gut bacteria are also deposited in the comb, but it has remained unknown which bacteria are deposited and whether distinct comb bacterial communities are sustained. Using high-throughput sequencing of the 16S rRNA gene, we explored the bacterial community compositions of 33 fungus comb samples from four termite species (three genera) collected at four South African geographic locations in 2011 and 2013. We identified 33 bacterial phyla, with Firmicutes, Bacteroidetes, Proteobacteria, Actinobacteria, and Candidate
\end{abstract}

Electronic supplementary material The online version of this article (doi:10.1007/s00248-015-0692-6) contains supplementary material, which is available to authorized users.

Saria Otani

Saria.Otani@bio.ku.dk

Lars H. Hansen

lhha@dmu.dk

Søren J. Sørensen

SJS@bio.ku.dk

Michael Poulsen

MPoulsen@bio.ku.dk

1 Department of Biology, Section for Ecology and Evolution, Centre for Social Evolution, University of Copenhagen, Universitetsparken 15, Building 3, 2100 Copenhagen East, Denmark

2 Department of Biology, Section for Microbiology, University of Copenhagen, Copenhagen, Denmark

3 Present address: Department of Environmental Science, Aarhus University, Aarhus, Denmark division TM7 jointly accounting for $92 \%$ of the reads. Analyses of gut microbiotas from 25 of the 33 colonies showed that dominant fungus comb taxa originate from the termite gut. While gut communities were consistent between 2011 and 2013, comb community compositions shifted over time. These shifts did not appear to be due to changes in the taxa present, but rather due to differences in the relative abundances of primarily gut-derived bacteria within fungus combs. This indicates that fungus comb microbiotas are largely termite species-specific due to major contributions from gut deposits and also that environment affects which gut bacteria dominate comb communities at a given point in time.

Keywords Macrotermitinae $\cdot$ Microbiota $\cdot$ Termitomyces . 16S rRNA sequencing $\cdot$ Symbiosis

\section{Introduction}

The Macrotermitinae subfamily of fungus-growing termites lives in an obligate symbiosis with a fungal mutualist in the genus Termitomyces [1]. Fungiculture in the Macrotermitinae evolved ca. 30 MYA, and the sub-family members have diversified to nearly 330 described species in 11 genera $[38,44]$. The adoption of the fungal ectosymbiont has been central for plant material decomposition [8, 43], allowing this termite lineage to become a major player in plant degradation and nutrient recycling in (sub)tropical Africa and Southeast Asia $[2,3]$. For example, up to $90 \%$ of all dry dead wood in Kenya is consumed by species of the Macrotermitinae [4]. This success has been attributed to the complementary mutualistic services of Termitomyces [5] and the microbial communities in the termite guts [6-8].

The most speciose genera of fungus-growing termites are Macrotermes, Odontotermes, and Microtermes, e.g., [5, 23]. 
Despite their importance, differences in their biology remain poorly understood, particularly in regard to what plant substrate is utilised for fungiculture. It has been proposed that Macrotermes species mainly ingest leaf litter $[18,21]$ and Odontotermes primarily wood and leaf litter [19, 20], while species of Microtermes have been described to be crop pests and wood feeders [45]. The nests of the three genera are structurally very different: Macrotermes mounds are conical and elevated with condensed shelving of fungus chambers on top of each other, with minimal soil separation between chambers (Fig. 1a). Odontotermes mounds are flatter and often have ventilation chimneys open to the environment. A main fungus comb is centralised within a deep cavity, and additional satellite fungus chambers surround this main comb [49, 50]. Microtermes species build large numbers of sub-spherical chambers that are connected with tunnels and often occur within other termite mounds [49, 50].

Colonies are highly organised with optimised division of labour between colony members to maintain the complex symbiotic system. Generally, a single reproductive pair (a queen and a king) starts the colony. The first workers forage for plant substrate and, in most Macrotermitinae genera, acquire the fungal symbionts from the surrounding habitat [9; exceptions being the genus Microtermes and the species Macrotermes bellicosus, which have vertical Termitomyces transmission]. As the colony matures, different castes are produced; older workers leave the colony to collect plant material, while young workers remain inside the nest [10]. These young workers inoculate the forage material by consuming it along with asexual spores of Termitomyces present in nutrientrich Termitomyces nodules [10]. After this first gut passage, the mixture is deposited as a sponge-like structure (the fungus comb) (Fig. 1a) $[5,11]$. This creates an upper darker part of the fungus comb that is the termite primary faeces (freshly deposited fungus comb) (Fig. 1a). The lighter (older) fungus comb, where new nodules are produced, is mainly fungal biomass after most plant decomposition has occurred (Fig. 1a) [12,13]. Older termites consume the oldest parts of the comb during a second gut passage $[10,14]$, after which only low concentrations of organic material are present in the resulting final faeces [15]. The plant substrate is thus efficiently digested via the combined efforts of fungal and gut bacterial enzymes $[8,16$, 17].

Recent molecular approaches has revealed a great diversity of primarily bacteria residing in fungus-growing termite guts $[16,17,34,46,47,51,52]$. We have recently described what appears to be a core microbiota of the Macrotermitinae: a set of 42 bacterial taxa shared between nine species of fungusgrowing termites [17]. This core is distinct from other nonfungus-growing termites $[16,17]$, but there is variation in the composition of gut microbiotas between fungus-growing termite species, suggesting potential co-adaptation between the termites and gut bacterial communities [17].
Because Macrotermitinae fungus combs are deposited gut contents, bacteria present in the gut could be transferred to the fresh comb. However, gut and fungus comb microbiotas from one macrotermitine species, Odontotermes formosanus, were analysed using pyrotag sequencing [24] and showed marked dissimilarities between worker hindgut and comb microbiotas, without detailed comparisons between $O$. formosanus guts and combs and without including comparison to other fungus-growing termite genera. To explore the composition of fungus comb microbiotas in more detail, we sampled 33 combs from three fungus-growing termite genera. Combs were collected from four different sites in South Africa over 2 years, and we used 16S rRNA 454 pyrosequencing to characterise communities. To test the hypothesis that comb communities are shaped by gut deposits, we performed $16 \mathrm{~S}$ rRNA Illumina MiSeq on worker guts from 25 of the same 33 nests. We determined which gut bacterial taxa are shared with fungus combs and explored whether unique comb taxa or taxa shared with guts have the biggest impact on microbiota fluctuations in comb compositions over time.

\section{Materials and Methods}

\section{Experimental Material, DNA Extractions, and Termite Species Identification}

\section{Fungus Combs}

Thirty-three fungus combs of three different fungus-growing termite genera were collected from four different sites [Experimental farm (Pretoria), RNC farm (Pretoria), Mookgophong, and Lajuma] in South Africa in February 2011 and February 2013 (Table 1; Fig. 1). In each case, a single apparently healthy comb (fresh and old comb) was collected and stored in RNAlater ${ }^{\circledR}$ (Ambion, USA). The FastDNA SPIN Kit for Soil (MP Biomedicals, USA) was used for fungus comb DNA extractions following the manufacturer's instructions, with the addition of two bead beating treatment steps for $25 \mathrm{~s}$ each at $6 \mathrm{~m} / \mathrm{s}$ using a FastPrep ${ }^{\text {TM }}$ Instrument (MP Biomedicals, USA) after the tissue lysis step, separated by 2 min of incubation on ice. DNA extracts were assessed spectrophotometrically using NanoDrop ND-1000 (Thermo Scientific, Germany).

\section{Termite Guts}

Twenty-five colonies from the 33 that were collected for the fungus combs were also used for termite sampling (Table 1, Fig. 1). Ten whole guts from major workers were dissected and pooled from each colony. The DNeasy Blood and Tissue Kit (Qiagen, Germany) was used for DNA extractions following the manufacturer's instructions. DNA yields were assessed 
Fig. 1 A Macrotermes natalensis fungus comb and the four sampling site locations. a A fungus comb contains two strata: the darker fresh fungus comb on the top and the lighter older comb below with less plant material and more Termitomyces biomass and nodules. b A map of South Africa with indications of the four collection sites in red dots, two of which were in Pretoria
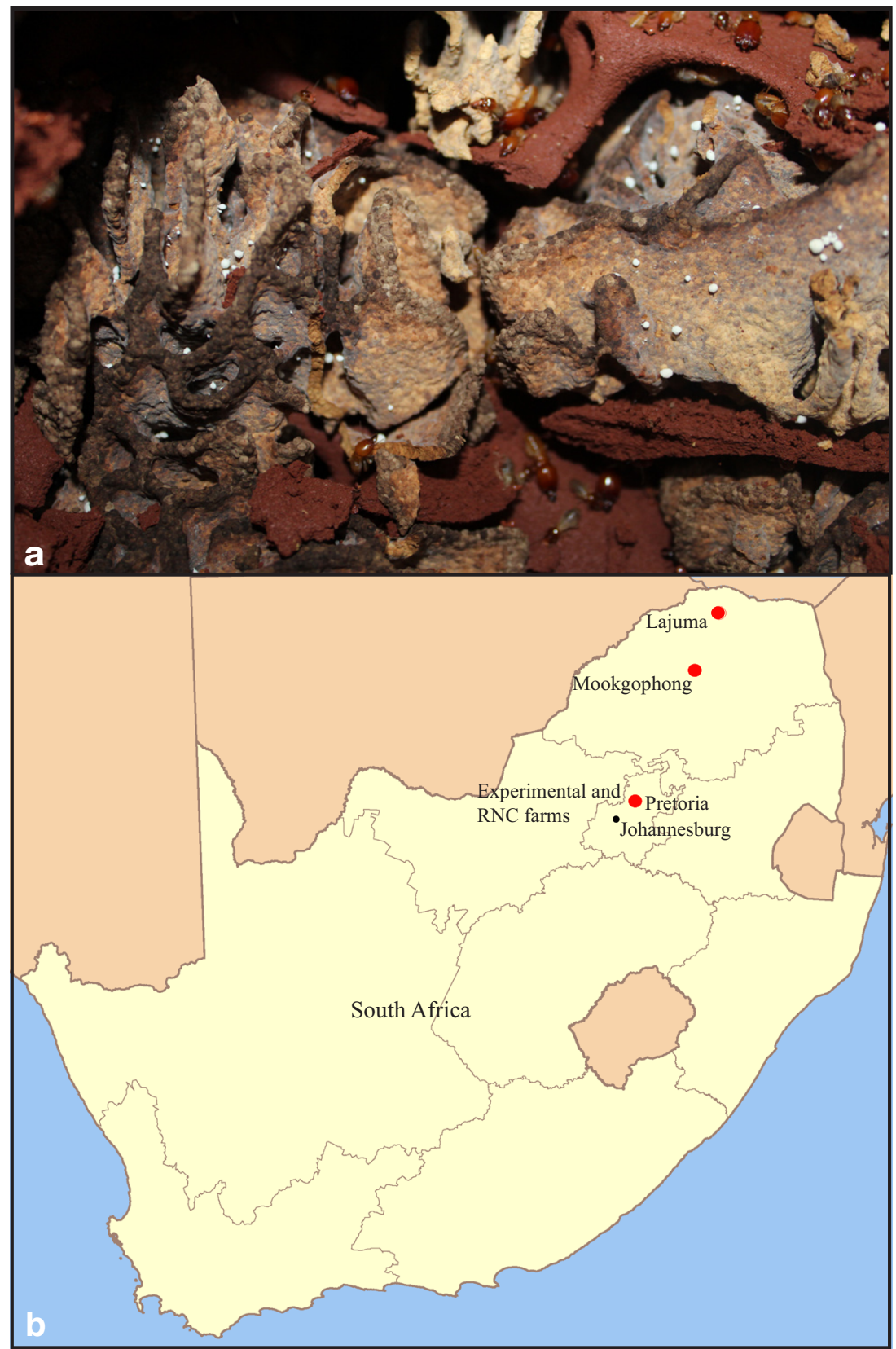

spectrophotometrically using NanoDrop ND-1000 (Thermo Scientific, Germany).

\section{Odontotermes Identification and Phylogenetic Analysis}

For Odontotermes colonies, in which molecular species determination was necessary due to uncertainty in morphological identification [26], workers and soldiers were collected and stored in $96 \%$ ethanol. This was not necessary for Macrotermes natalensis, because only a single Macrotermes species exists in this area [25], and was not done for Microtermes, because termites could not be obtained for Microtermes samples from 2011. The DNeasy Blood and
Tissue Kit (Qiagen, Germany) was used after a homogenisation step to extract DNA from Odontotermes termite heads. PCR was performed on 14 Odontotermes DNA extracts to amplify the mitochondrial cytochrome oxidase II gene (COII) to obtain a barcode that could be compared to available sequences in GenBank. Reactions were prepared in $25 \mu \mathrm{l}$ final volume using A-tLeu forward primer (5'-ATG GCA GAT TAG TGC AAT GG-3') and B-tLys reverse (5'-GTT TAA GAG ACC AGT ACT TG-3') [27, 28]. The PCR mixture contained $8.5 \mu$ sterile distilled water, $1 \mu \mathrm{l}$ of each primer, $2 \mu \mathrm{l}$ template, and $12.5 \mu \mathrm{l}$ REDTaq ReadyMix (SigmaAldrich, USA). The conditions for PCR were $98{ }^{\circ} \mathrm{C}$ for $30 \mathrm{~s}$ followed by 35 cycles of $94{ }^{\circ} \mathrm{C}$ for $30 \mathrm{~s}, 50{ }^{\circ} \mathrm{C}$ for $30 \mathrm{~s}$, and 


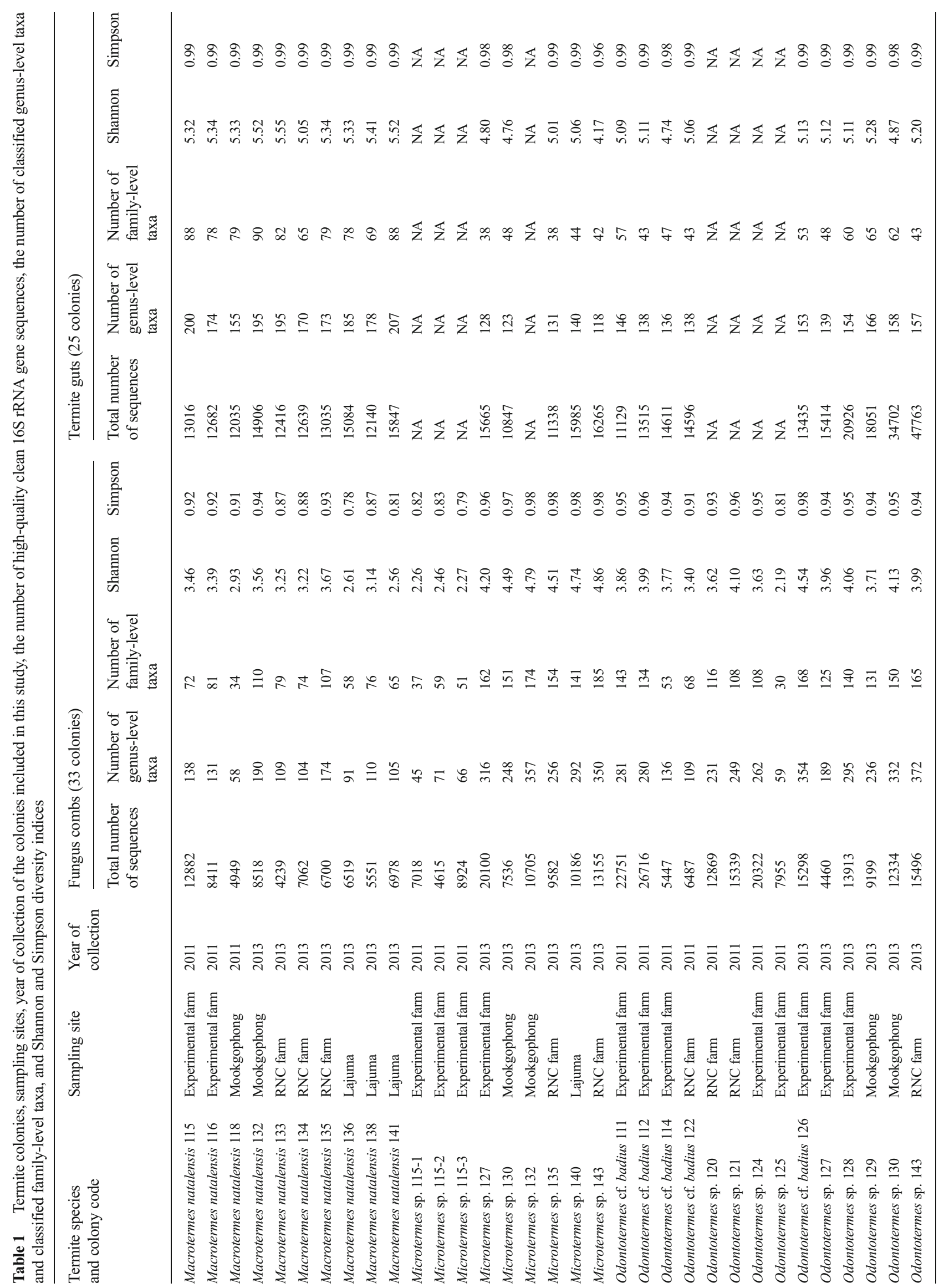


$72{ }^{\circ} \mathrm{C}$ for $30 \mathrm{~s}$ with a final extension step at $72{ }^{\circ} \mathrm{C}$ for $4 \mathrm{~min}$. Target PCR products were visualised by agarose gel electrophoresis and purified using MSB Spin PCRapace (STRATEC Molecular, Germany). The samples were subjected to sequencing at Eurofins MWG Operon (Ebersberg, Germany). Resulting sequences were aligned in Geneious 6.1.6 using the MUSCLE algorithm [29], and a Neighbour Joining tree, including the 14 Odontotermes colonies involved in the present study, two additional colonies, and Odontotermes COII sequences available from GenBank, was generated in TreeView [30] with Kimura two-parameter estimates. Sequences generated as part of this study have been deposited in GenBank (accession numbers KJ4590682-KJ4590697).

\section{Bacterial Community Characterisations in Fungus Combs}

\section{Fungus Comb PCR Amplification and 454 Pyrotag Sequencing}

The 16S rRNA gene was amplified using the primers $341 \mathrm{~F}$ (5'-CCTACGGGRBGCASCAG-3') and 806R (5'GGACTACNNGGGTATCTAAT-3') flanking the hypervariable V3-V4 regions [31]. The primers were modified by adding sample-specific multiplex identifier barcodes (MID) (5'-Adaptor A) to the forward primer and a universal sequence ( $5^{\prime}$-Adaptor B) to the reverse primer. The amplification reaction was prepared in $20 \mu \mathrm{l}$ final volume containing the following: $12.4 \mu \mathrm{l}$ sterile distilled water, $0.4 \mu \mathrm{l}$ dNTPs $(10 \mu \mathrm{M}), 4 \mu \mathrm{l}$ $5 \times$ HF buffer, $1 \mu \mathrm{l}$ of each primer, $1 \mu \mathrm{l}$ template, and $0.2 \mu \mathrm{l}$ Phusion High-Fidelity DNA Polymerase (Thermo Scientific, Germany). The conditions for PCR were $98{ }^{\circ} \mathrm{C}$ for $30 \mathrm{~s}$ followed by 15 cycles of $98{ }^{\circ} \mathrm{C}$ for $5 \mathrm{~s}, 56^{\circ} \mathrm{C}$ for $20 \mathrm{~s}$, and $72{ }^{\circ} \mathrm{C}$ for $20 \mathrm{~s}$ with a final extension step at $72{ }^{\circ} \mathrm{C}$ for $5 \mathrm{~min}$. This reaction was done after a previous amplification using the same primers and conditions but with 30 PCR cycles. The final target PCR products were visualised by agarose gel electrophoresis, then extracted and purified from the gel using Montage DNA Gel Extraction Kit (Millipore Corporation, USA). DNA concentrations were quantified using Quant-iT dsDNA High-Sensitivity Assay Kit and Qubit fluorometer (Invitrogen). The samples were subjected to sequencing on GS FLX Titanium PicoTiterPlate using a GS FLX Titanium Sequencing Kit according to the manufacturer's instructions (Roche).

\section{Sequence Filtering and Taxa Classification}

The raw flowgrams were fed into MOTHUR (version 1.31.2, [32]), where multiplexed reads were assigned to samples based on unique barcodes and erroneous reads were removed by denoising. Sequences containing ambiguous bases $(\mathrm{N})$, with mismatches to the 16S rRNA primers, homopolymer stretches longer than 10 bases, or sequences shorter than $200 \mathrm{bp}$, were excluded from subsequent analyses during several filtering steps in MOTHUR. Clean sequences were submitted to the Sequence Read Archive (SRA) in GenBank (accession numbers SRR1293514-SRR1293516, SRR1293655, SRR1293679, SRR1293686, SRR1293696-SRR1293703, SRR1293771, SRR1293794, SRR1293811-SRR1293813, SRR1293816-SRR1293818, SRR1293820-SRR1293825, SRR1293827, SRR1293828, SRR1293831, SRR1293837, SRR1293845). High-quality sequences were aligned against the SILVA 102 non-redundant database using MOTHUR. Alignments were subsequently assigned to taxa using the naïve Bayesian classifier with a confidence threshold of $60 \%$ and a manually curated reference database DictDb v. 2.3 [33]. This database was generated from the SILVA database with additional termite and cockroach gut 16S rRNA gene sequences added to improve the classification resolution; it is available upon request. Rarefaction curves based on $97 \%$ sequence similarity cutoff were generated using R [40].

\section{Comparative Analyses of Community Diversity and Similarity}

The representative clusters were sorted according to the genus-level classifications, and taxa abundances were calculated as the number of reads per taxon. R [40] was used to calculate community similarities; principal coordinates analysis (PCoA) was performed based on the Bray-Curtis index to determine community similarity between all 33 samples. Three additional PCoA analyses were performed on samples from within each of the three termite genera.

\section{Bacterial Community Characterisations in Termite Guts}

\section{Termite Worker Gut PCR Amplification and MiSeq Sequencing}

The V4 region of the 16S rRNA gene was amplified using the primers v4.SA504 (5'-AATGATACGGCGAC CACCGAGATCTACACCTGCGTGTTATGGTAATTGTGTGCCAGCMGCCGCGGTAA-3') and v4.SB711 (5'CAAGCAGAAGACGGCATACGAGATTCAGCGTTAGTCAGTCAGCCGGACTACHVGGGTWTCTAAT-3'). The V4 region amplification was done using a dual-indexing sequencing strategy (41), and the PCR mixture was prepared in $20 \mu \mathrm{l}$ volume containing $11.85 \mu \mathrm{l}$ sterile distilled water, $2 \mu \mathrm{l}$ of each primer $(4.0 \mu \mathrm{M}), 2 \mu \mathrm{l}$ of $10 \times$ AccuPrime PCR buffer II (Life Technologies, USA), $1 \mu$ l DNA template, and $0.15 \mu \mathrm{l}$ AccuPrime High Fidelity Taq DNA polymerase (Life Technologies, USA). PCR conditions were $95^{\circ} \mathrm{C}$ for $2 \mathrm{~min}$ followed by 30 cycles of $95{ }^{\circ} \mathrm{C}$ for $20 \mathrm{~s}, 55^{\circ} \mathrm{C}$ for $15 \mathrm{~s}$, and $72{ }^{\circ} \mathrm{C}$ for $5 \mathrm{~min}$ followed by $72{ }^{\circ} \mathrm{C}$ for $10 \mathrm{~min}$ (42). Library normalisation was done using Life Technologies SequalPrep Normalization Plate Kit (Life Technologies, USA) following 
the manufacturer's instructions. Sample concentration was measured using Kapa Biosystems Library Quantification kit for Illumina platforms (Kapa Biosystems, USA). The size of the library amplicons was determined using the Agilent Bioanalyser High Sensitivity DNA analysis kit (Invitrogen). The samples were subjected to sequencing on the Illumina MiSeq platform using MiSeq Reagent Kit V2 500 cycles (Illumina) [41, 42].

\section{Sequence Filtering and Taxa Classification}

Sequence analysis was performed using MOTHUR (version $1.34 .3,[32]$ ), and the standard operating procedure (SOP) was followed as described at http://www.mothur.org/wiki/MiSeq SOP [41]. Briefly, the paired end reads were assembled into contigs and then sequences were subjected to several filtering steps. Clean reads were submitted to SRA, GenBank (accession numbers SRR2085096-SRR2085120). High quality sequences were aligned against the SILVA 102 database. Alignments were afterwards assigned to taxa with a confidence threshold of $80 \%$, and operational taxonomic units (OTUs) were calculated at $3 \%$ species level classification. Finally, rarefaction curves based on $97 \%$ sequence similarity cutoff were generated using R [40].

\section{Comparative Analyses of Gut Community Diversity and Similarity}

Relative abundances of identified taxa were calculated as the number of reads per taxon for the 25 gut communities. PCoA was performed in $\mathrm{R}$ [40] based on the Bray-Curtis index to determine community similarity between the 25 samples from the four termite species from the three termite genera (M. natalensis, Microtermes sp., and Odontotermes cf. badius and Odontotermes sp.).

\section{Comparison of Comb and Gut Community Compositions}

To investigate bacterial community similarities between the guts and fungus combs, we identified overlapping and unique taxa in each of the 25 colonies with sequencing data from both worker guts and fungus combs. Quantitative contributions of the overlapping taxa were calculated as the proportion of reads that were assigned to overlapping versus unique taxa in guts and combs. The use of different sequencing technologies and primer sets to amplify the V4 region of the 16S rRNA gene in fungus combs (454 titanium sequencing) and guts (Illumina MiSeq) precluded comparisons to the genus level, so all comparisons were performed at the family level. Gut and comb family bacterial taxa were compared manually and separately for each of the 25 tested colonies, and identical taxonomical names were considered shared taxa. Sequence comparisons were not possible due to length differences between 454 and MiSeq sequences (http://www.mothur.org/).

To evaluate whether shared or unique bacterial taxa contributed the most to similarities between communities within and between termite species across years, we performed four additional PCoAs. Two of these PCoAs compared fungus comb communities: one included only taxa that were also present in guts and the other included only taxa that were unique to combs. The remaining two PCoAs compared gut communities: one included taxa that were also present in combs and the other included only taxa that were unique to guts.

\section{Results}

\section{Odontotermes Phylogenetic Analysis}

COII genes were successfully amplified from the 14 Odontotermes samples, in addition to two samples not included in this study. BLAST (Table S1) and phylogenetic analysis (Fig. S1) of the 390-bp fragment suggested that we had collected five Odontotermes cf. badius and nine Odontotermes sp. colonies from a well-supported phylogenetic clade most closely related to Odontotermes sp. and Odontotermes latericeus (Fig. S1). Therefore, combs from this species are hereafter labelled Odontotermes sp.

\section{Pyrosequencing Data from Fungus Combs}

We obtained between 4239 and 26,716 high-quality reads (average \pm SE 10,673 \pm 977 ) per comb sample (Table 1). The resulting rarefaction curves were approaching saturation for most samples, indicating that coverage in general was sufficient for community structure analyses. Even though the rarefaction curves for the samples Odontotermes sp. 127, Odontotermes sp. 129, and Microtermes spp. 135 indicated that additional sequencing would be required to cover the expected bacterial diversity (Fig. S2), they were included in the analyses. The number of bacterial genera ranged between 58 and 372 (average 196) (Table 1), with M. natalensis combs harbouring the least (M. natalensis 121, Microtermes sp. 222, Odontotermes sp. 229, and O. cf. badius 232). Samples collected in 2011 had fewer bacterial taxa, particularly for Microtermes sp., while sampling site did not appear to influence the observed number of taxa in combs (Table 1). Shannon and Simpson diversity indices were similar across the comb samples with slightly higher values in Microtermes comb communities from 2013 (Table 1).

\section{Taxonomic Composition of Comb Bacterial Communities}

Using the termite-specific improved SILVA database (DictDb) allowed relatively high classification success in all samples 
(54.5-76.8 \% at the genus level; Table 1). Thirty-three bacterial phyla were identified in the $33 \mathrm{combs}$, and the five most abundant phyla accounted for $92.4 \%$ of the total number of reads (Firmicutes, Bacteroidetes, Proteobacteria, Actinobacteria, and Candidate division TM7) (Fig. S3, Table S2). Among the most abundant phyla, Actinobacteria were more abundant in Microtermes sp. compared to M. natalensis and Odontotermes, whereas Firmicutes and Bacteroidetes were less abundant in Microtermes sp. (Fig. S3). Among the less abundant phyla, Acidobacteria and Chloroflexi were more abundant in Microtermes sp. from 2013 compared 2011 and to the other termite genera. Spirochaetes were not abundant in combs, representing on average only $1.2 \%$ of the total classified reads (Fig. S3, Table S2).

Most bacterial genera were unevenly distributed across combs. An overview of all 1795 genus-level taxa obtained in the analysis can be found in the interactive Table S2, which provides read percentages for each bacterial taxon from phylum to genus level for each comb sample. Of these genus-level taxa, 1387 were bacteria, and the 100 most abundant bacteria across all 33 combs are presented in Fig. S5 as a heatmap of relative abundances. Among these, only a few taxa were present in relatively high abundance across all comb samples; for example, Alistipes (13.3\% average abundance across all combs), BCf917 termite group in the family Rikenellaceae (3.6\%) and Herbaspirillum 1 in the Oxalobacteraceae (2.2\%). A number of other genera were abundant on average, but absent or in low abundance in some combs; for example, uncultured 1 in the family Corynebacteriaceae $(6.1 \%)$, uncultured 3 in the family Peptostreptococcaceae (3.9\%), Gut Cluster 1 in the family Ruminococcaceae (2.3\%), Incertae Sedis 2 in the Planococcaceae (2\%), Gut Cluster 2 in the family Lachnospiraceae (1.9 \%), "Candidatus Arthromitus" (1.7\%) and Incertae Sedis 6 (Planococcaceae 2) (1.6\%) (Table S2). Despite the presence of several abundant taxa in the communities, the appreciable difference between the 2 years of collection led to a large variation in relative abundances between fungus combs within and between genera (Table S2, Fig. S5). For example, the dominant taxon Alistipes was less abundant in Microtermes combs in 2011 compared to 2013, uncultured 1 (Corynebacteriaceae) was present only in $M$. natalensis and Microtermes sp. samples from 2011 (particularly M. natalensis 118), and uncultured 3 (Peptostreptococcaceae) was only detected in three samples (M. natalensis 118 , Odontotermes sp. 124, and Odontotermes sp. 125) from 2011 (Fig. S5, Table S2). Similarly, Gut Cluster 2 and "Candidatus Arthromitus" were detected in high abundance only in 2013, while Herbaspirillum 1 was only abundant in M. natalensis and Microtermes sp. from 2011. The BCf917 termite group was relatively abundant in $M$. natalensis samples in 2013, less in Odontotermes in 2011, and in very low relative abundance in the remaining samples (Fig. S5, Table S2). Incertae Sedis 2 and Insertae Sedis 6 showed high relative abundances only in
M. natalensis 118 , Odontotermes $\mathrm{sp} .124$, and Odontotermes sp. 125 from 2011 (Fig. S5, Table S2).

\section{Comb Ordination Analyses}

We visualised Bray-Curtis distances between communities in four different PCoAs: the entire data set (33 samples, Fig. 2a), M. natalensis (10 fungus combs, Fig. S4B), Microtermes sp. ( 9 fungus combs, Fig. S4C), and $O$. cf. badius and Odontotermes sp. (14 fungus combs, Fig. S4A). There was a distinct separation in all PCoAs between years (Figs. 2a and S4), which was also evident for individual termite genera (Fig. S4A-C). Community compositions were secondarily influenced by termite species origin, with bacterial communities of combs collected from a termite species being more similar to each other than to samples collected from other termite species in the same year (Fig. 2a). Within termite species, there was also an apparent effect of sampling site; for example, the two Odontotermes sp. colonies collected at the RNC farm in 2011 were distant from the remaining colonies of Odontotermes sp. collected at other locations (Fig. S4A).

The $O$. cf. badius 122 comb community was more similar to samples from the other Odontotermes species from the same sampling site (RNC farm) than to samples from other sites (Fig. S4A). M. natalensis showed the same trend, with M. natalensis 115 and 116 fungus combs collected in 2011 at the Experimental farm being more similar to each other than to M. natalensis 118 collected the same year in Mookgophong (Figs. 2a and S4B), and Lajuma-collected M. natalensis samples from 2013 clustering closer to each other than to communities from other sites (Figs. 2a and S4B). However, some combs from the same termite species (e.g. Mn138 and Mn134, Od128, and Od130) were similar in composition despite being from geographically distinct sites up to $450 \mathrm{~km}$ apart. A spatial effect of sampling site did not appear for Microtermes fungus comb communities, probably due to the limited number of samples from 2011 (Table 1, Fig. S4C).

\section{Illumina MiSeq Data from Worker Guts}

16S rRNA gene sequencing of the guts generated between 10 , 847 and 47,763 high-quality reads (average \pm SE 16,321 \pm 1612) per gut sample (Table 1), and rarefaction analysis showed sufficient coverage of bacterial communities (Fig. S6). A total of 5178 unique OTUs at the $3 \%$ cutoff level were identified after filtering and sequence analysis of gut communities, with 425 to 860 OTUs per gut sample (Table 1).

\section{Taxonomic Composition of Gut Bacterial Communities}

Gut community reads were classified using the publicly available SILVA database, and 27 bacterial phyla were identified in 
the 25 termite guts (Fig. S7). We were not able to classify the gut sequences using the termite specific DictDb v. 2.3 database, which was used for the comb reads, as it was developed for 454 pyrosequencing analyses. The most abundant phyla were Bacteroidetes, Firmicutes, Spirochaetes, Proteobacteria, and Planctomycetes, which accounted for $93.5 \%$ of all sequence reads (Fig. S7, Table S3). No differences in phylum relative abundances were noticed between the samples from different years (2011 and 2013), whereas differences were apparent between guts from different termite genera (Fig. S7). For example, Spirochaetes was more abundant in Microtermes and Odontotermes compared to Macrotermes (Table S3) and Planctomycetes was less abundant in Microtermes guts compared to the other two genera (Table S3). Members of Actinobacteria were generally low in abundance and accounted for only $2.1 \%$ of the total number of reads.

Genus-level OTU classification identified 495 bacterial genera from the 25 gut communities. An interactive table of all generated OTUs and their assigned taxa is presented in Table S3 with the corresponding relative abundances. A heatmap of the 100 most abundant gut bacteria is presented in Fig. S8. Alistipes was the most abundant (on average $13.2 \%$ across all guts), followed by Treponema $(9.1 \%)$, and Dysgonomonas (7.1\%). Unlike comb communities, relative abundances of bacterial taxa were not different between 2011 and 2013 gut samples (Fig. S8, Table S3), whereas differences were apparent between termite host genera and species. For example, Treponema was more abundant in Odontotermes and Microtermes guts (on average 6.3 and $3.6 \%$, respectively) compared to Macrotermes guts (1.2 \%) (Fig. S8, Table S3), and the genus Parabacteroides was high in abundance in Odontotermes guts (on average $3.4 \%$ ), particularly in $O$. cf. badius, compared to Macrotermes (0.2 \%) and Microtermes $(0.07 \%)$ (Fig. S8, Table S3). Tannerella was abundant in $O$. cf. badius guts ( $2.9 \%)$, but almost absent in Odontotermes sp. guts $(0.002 \%)$ (Fig. S8, Table S3).

\section{Gut Ordination Analyses}

Gut microbiota similarities were assessed by Bray-Curtis distances and in PCoA plots (Fig. b). A clear signal of termite species was present in gut communities, where microbiotas within a termite species were more similar to each other than to other termite species (Fig. 2b). In contrast to comb communities, there was no effect of year on gut bacterial composition (Fig. 2b).

\section{Comparison of Comb and Gut Community Compositions}

The comparison between gut-associated and comb-associated microbiotas was done separately for each of the 25 colonies (Fig. 3). There were between 14-48 bacterial families overlapping between the guts and fungus combs in the 25 colonies
(Fig. 3). In Microtermes, $36.8-73.8 \%$ of families identified in guts were present in combs, $26.6-53.3 \%$ of gut families were present in Macrotermes combs and 40.4-80.7\% of Odontotermes gut families were present in combs. Smaller proportions of families identified in the combs were present in guts as follows: $8.6-22.5 \%$ in Microtermes, $36.5-61.8 \%$ in Macrotermes, and 17.6-35.9\% in Odontotermes (Venn diagrams in Fig. 3; Table S4). Thus, Microtermes and Odontotermes colonies had more overlapping families between guts and fungus combs and contained relatively few unique gut families compared to M. natalensis (Fig. 3). The proportion of reads belonging to overlapping families ranged from 64.6 to $98 \%$ out of the total number of gut reads across colonies (64.6-97.7 \% in Microtermes, 79.7-92.5\% in Macrotermes, and 77-98 \% in Odontotermes), and 31.9$96.3 \%$ of the total number of fungus comb reads across colonies were shared with guts (31.9-73.7\% in Microtermes, $58.5-96.3 \%$ in Macrotermes, and $51.9-89.5 \%$ in Odontotermes) (bars in Fig. 3, Table S4). Thus, while the reads of shared families dominated gut communities in all termite genera, the proportion of shared comb reads varied by termite genus. In $M$. natalensis, shared reads dominated both gut and comb communities, while larger portions of Microtermes and Odontotermes comb communities contained reads from families not present in gut communities (bars in Fig. 3, Table S4).

To investigate whether unique or overlapping bacteria contributed the most to the differences between fungus combs across years, Bray-Curtis distances (including bacterial relative abundance) were calculated and visualised in four PCoAs that included taxa in (1) comb communities that overlapped with guts (Fig. 4a), (2) gut communities that overlapped with combs (Fig. 4b), (3) comb communities that did not overlap with guts (Fig. 4c), and (4) gut communities that did not overlap with combs (Fig. 4d). The first of these (Fig. 4a) showed the same temporal separation between years as the complete fungus comb microbiotas (Figs. 2a and 4a). Similarly, analysis of the gut bacteria overlapping with fungus combs separated in PCoA space (Fig. 4b) in a similar pattern as when analysing the entire gut microbiotas in Fig. 2b, except that the two Odontotermes spp. were close in PCoA space. These patterns of community similarities were diluted when only unique gut or comb bacteria were included in the analyses (Fig. 4c, d). Nevertheless, comb communities remained largely separated by year, except in Macrotermes (Fig. 4c), while gut communities remained separated by termite species, but with substantially increased variation within species (Fig. 4d).

\section{Discussion}

We present the first detailed comparison of bacterial communities in guts and fungus combs of fungus-growing termites. 


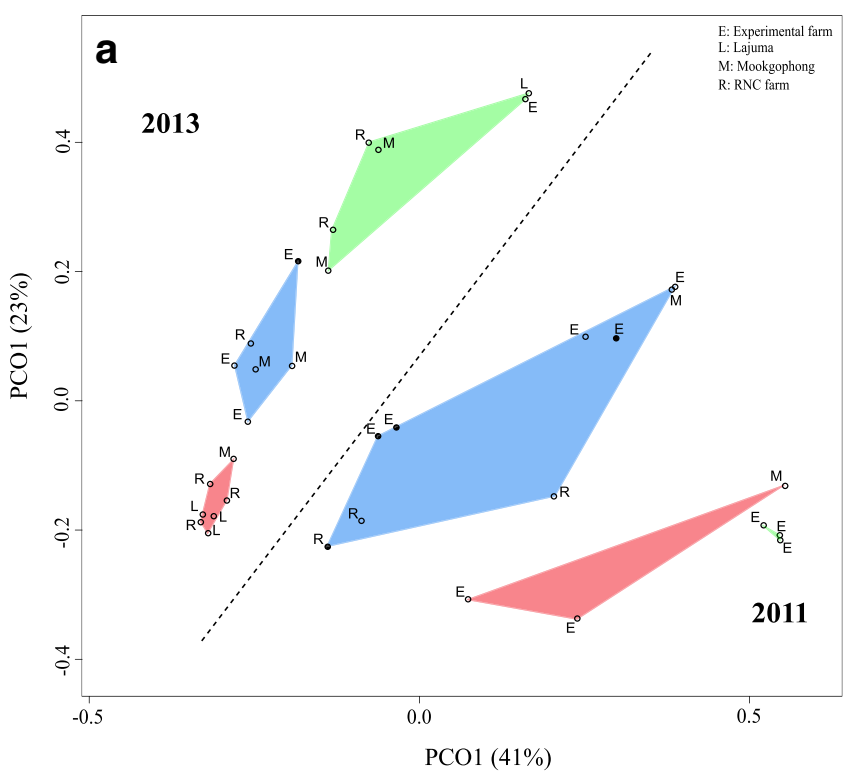

Fig. 2 a PCoA similarity analysis of the 33 fungus comb samples visualised via Bray-Curtis distances across samples. Fungus comb samples from the same host taxa are connected and have the same colour (red: Macrotermes natalensis, blue: Odontotermes, solid circles are $O$. cf. badius and open circles are Odontotermes sp., and green:

By comparing 33 different comb microbiotas from within and between species from three termite genera over 2 years, we found that communities were often very similar within termite species when obtained from the same year, but that there were marked shifts in comb community compositions across years of sampling. In contrast, analyses of guts from 25 of the 33 colonies showed that gut communities were consistent in composition within termite host species over time. Comparisons of comb and gut communities showed that (i) a proportion of bacterial families are shared between guts and fungus combs, (ii) in most colonies, the majority of reads in comb communities belong to families shared with gut microbiotas, (iii) unique families are present in both guts and combs, but these represent a minority of sequence reads, and (iv) although the above patterns were true across the three termite genera (four species) tested, there were consistent differences between termite species. Below, we discuss the implications of these findings, focusing on how bacterial communities of fungus combs are assembled.

\section{Shared Bacteria Across Combs and Guts}

Comb communities were dominated by bacteria that were also present in termite guts (Fig. 3). Because gut and fungus comb communities were sequenced using two different sequencing platforms, different primer sets, bioinformatics analyses [41] and reference databases, detailed genus-level community analyses were not possible and comparisons were restricted to higher phylogenetic levels. However, checking the two sets of primers using the SILVA TestPrime online tool (http://www.

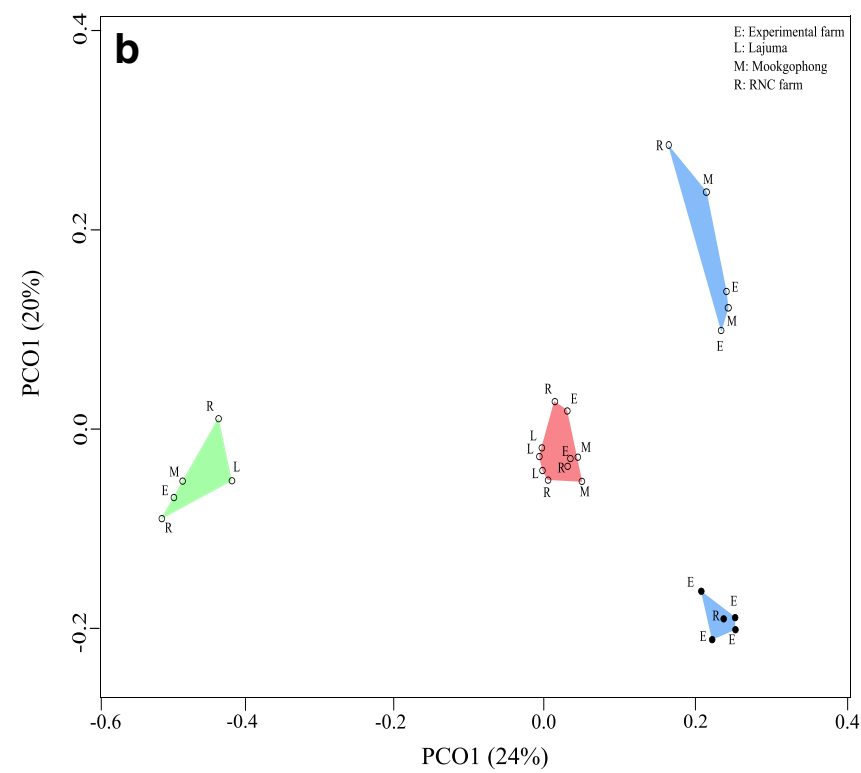

Microtermes sp.). Sampling locations are indicated, and the dashed line separates the sampling years 2011 and 2013. b PCoA similarity analysis of the 25 gut samples visualised via Bray-Curtis distances across samples. Gut samples from the same host taxa are connected and coloured as in a

arb-silva.de/search/testprime/) indicated that they should not be biased towards or against the major phyla identified in either of the two analyses.

Firmicutes and Bacteroidetes dominated both guts and fungus combs and accounted for $60 \%$ of all reads, which is comparable to previous reports on fungus-growing termite guts $[16,17]$. These phyla include members that are important for carbohydrate metabolism, reductive acetogenesis, and fungal cell wall degradation [43, 48]. Actinobacteria were more abundant in combs compared to guts (Figs. S3 and S7), suggesting that growth conditions for Actinobacteria are better in combs than in guts. It could also indicate that they are introduced to the comb from the surrounding soil, where Actinobacteria are abundant [52]. It has been suggested that Actinobacteria may play defensive roles in the symbiosis by suppressing unwanted microorganisms [39]. If this is so, our findings suggest that this function may be more important in fungus combs than in termite guts. The remaining phyla were similar in relative abundances between the guts and combs (Figs. S3 and S7).

A comparison of guts and combs at the level of bacterial families (Fig. 3) showed similar overall patterns of shared communities across the four species (three genera) of termites. Large fractions of sequence reads were consistently shared between combs and guts within colonies (bars in Fig. 3), implying that when fresh comb is built through gut deposits of fragmented substrate inoculated with Termitomyces, this is accompanied by the deposition of a substantial diversity of gut bacteria. These deposits are likely to contain a large proportion of termite gut symbionts, but it is conceivable that they may also include 

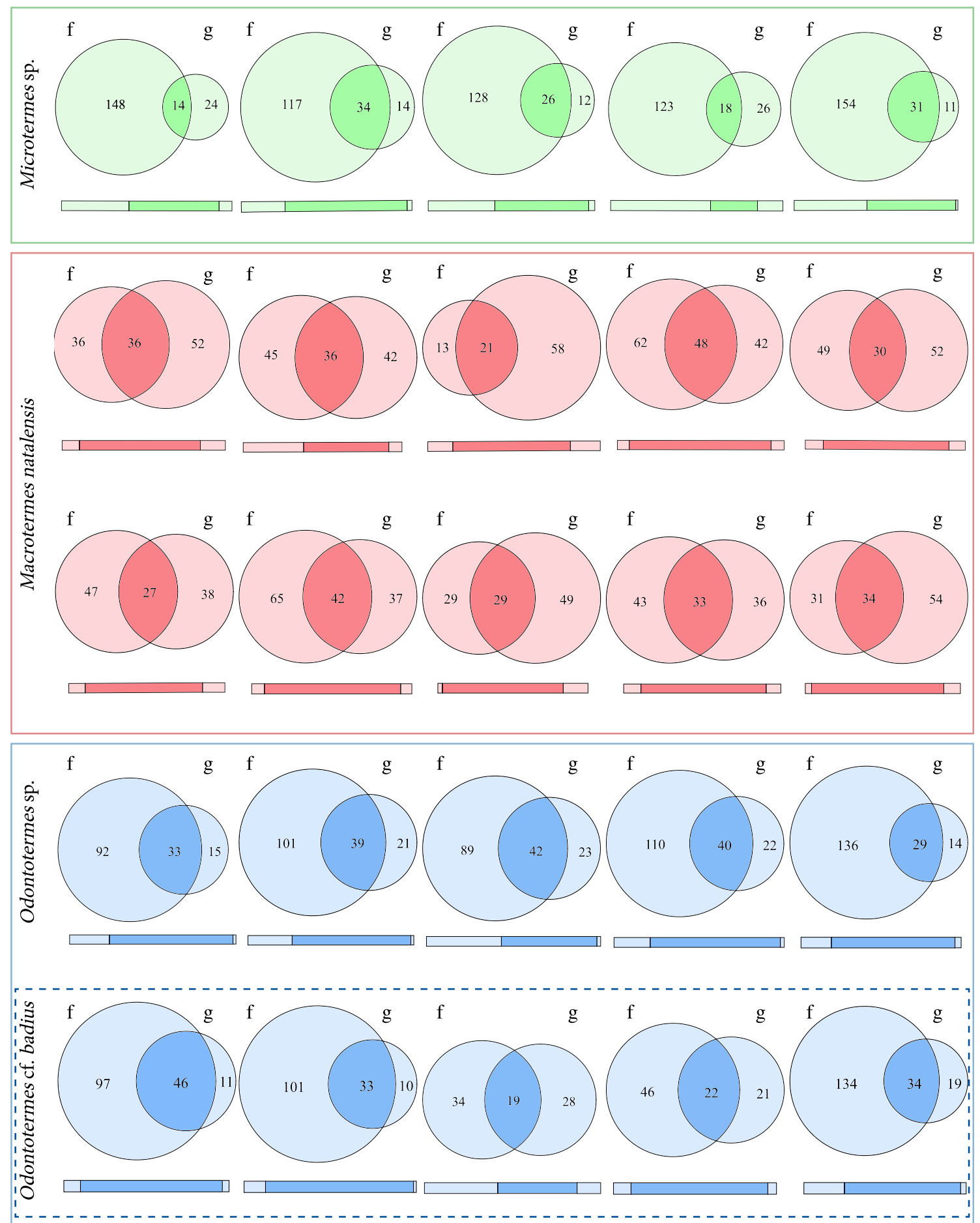

Fig. 3 Venn diagrams of shared fungus comb and gut microbiota families in the 25 colonies where samples for both could be sequenced. $f$ are the fungus comb communities and $g$ are the gut communities. Colonies from the same host taxa have the same colour (red: Macrotermes natalensis, green: Microtermes sp., and blue: Odontotermes spp. (O. cf. badius within dashed box)). The numbers represent the number of family-level bacterial taxa identified in the

substrate microorganisms that pass through the termite gut. Although we cannot distinguish between these two sources, fungus combs only, in the guts only, or shared between the two communities. The bars represent the abundances of the high-quality reads that were assigned to bacterial families presented in the Venn diagrams in each colony (light colour: proportion of reads that are unique to guts or fungus combs, dark colour: proportion of reads shared by the two communities). Detailed numbers are presented in Table S4

the former is likely to be the most important, as fungus combs contain the dominant gut symbionts of fungus-growing termites. 

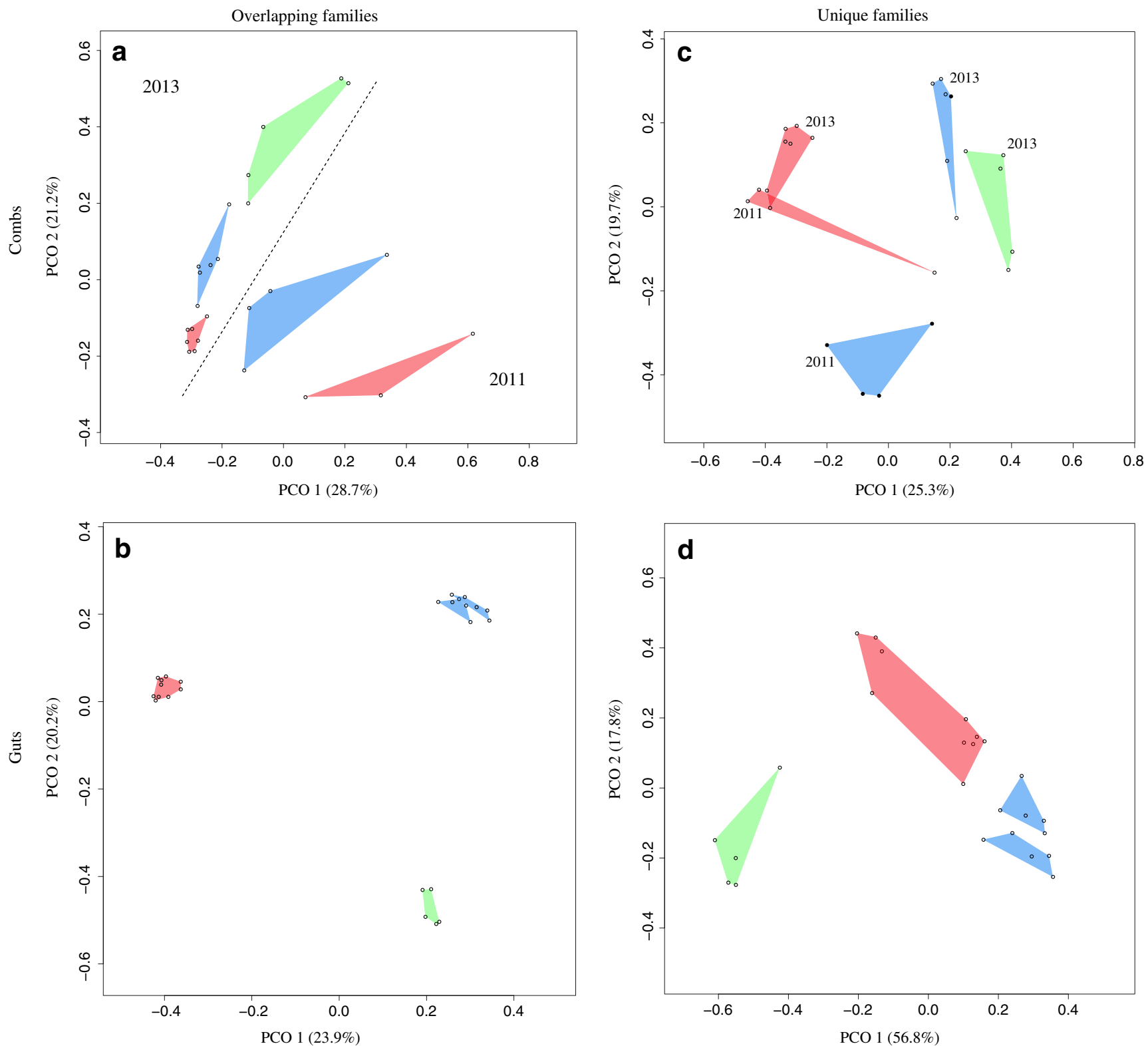

Fig. 4 PCoA similarity analyses visualising Bray-Curtis distances between fungus comb microbiotas (a, c) and gut microbiotas (b, d) when measuring distances between fungus comb bacterial communities that overlap with the gut bacteria (a), distances between fungus comb bacterial communities that are unique to combs (c), distances between gut bacterial communities that overlap with fungus comb bacteria (b), and

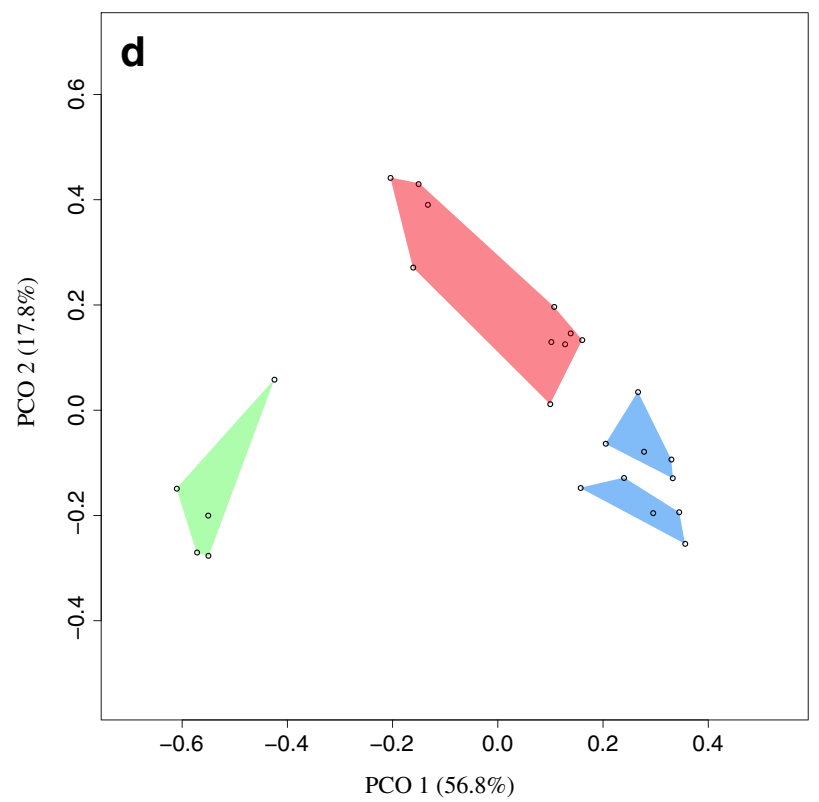

distances between gut bacterial communities that are unique to the guts. Samples from the same host taxa are connected and have the same colour (red: Macrotermes natalensis, green: Microtermes sp., blue: Odontotermes spp., solid circles are $O$. cf. badius and open circles are Odontotermes sp.)

Previous studies have suggested that diet may influence the composition of termite gut microbiota [35, 36], and different fungus-growing termite genera tend to use different substrates for fungiculture [18-22]. Our finding of consistent gut microbiota compositions over time and geographical distances suggests that gut communities are resilient to changes in substrate microbial content; however, different substrates may transport different bacteria to the combs if these bacteria remain low in abundance within guts, but proliferate after deposition in combs. At present, substrate use and the microbial content

of substrates remain poorly characterised, and further work will be needed to establish which bacteria are potentially delivered to combs through the substrate.

Although most sequence reads belonged to shared bacterial families, several taxa were unique in guts or fungus combs. Unique gut taxa are likely to be symbionts that are not deposited with the fresh comb, e.g. symbionts in low abundance within guts or symbionts that are tightly associated with the termite gut wall and absent in the lumen. Unique comb taxa are likely to be from the surrounding soil or substrate-dwelling 
bacteria that pass the gut and only proliferate within combs. In a recent study, Makonde et al. [52] detected increased relative abundances of Actinobacteria in the mounds and savannah soils compared to termite guts in two fungus-growing termite genera. However, since soil or mound samples were not available for comparison in this study, we could not test their contributions to the identified unique bacterial taxa in combs.

\section{Factors Contributing to Differences in Comb Communities Between Termite Genera}

Both gut and comb communities consistently cluster according to termite host species (Fig. 2a, b), making termite taxon the main predictor of comb community structure. The differences in gut microbiotas between termite species, and particularly termite genera $[16,17$, this study], and the presence of many gut taxa in fungus combs imply that differences in comb microbiotas between termite genera are shaped by differences in gut deposits between termite genera (Fig. 2).

The variation in the shared bacterial communities between guts and fungus combs appears to be related to variation in the total number of bacterial families in the guts and fungus combs between the termite genera. For example, Macrotermes colonies harboured the highest number of gut families (on average 80), but the lowest number of comb bacterial families (on average 76) (Table 1). It is likely due to this variation between Macrotermes gut and comb bacterial counts that fewer gut families are shared with fungus combs, while more comb families are present in guts compared to the other two termite genera (Table 1, Fig. 3). In contrast, most gut families in Microtermes and Odontotermes were also present within combs, but fewer fungus comb families were present in guts (Fig. 3, Table S4). However, when adding the number of sequence reads assigned to the families shared between guts and fungus combs, most reads from Macrotermes guts and combs are from shared bacterial families (bars in Fig. 3, Table S4, see "Results" section), while Microtermes and Odontotermes share most gut sequence reads with fungus combs, and relatively smaller proportions of their fungus comb reads overlap with guts (particularly in Microtermes) (bars in Fig. 3, Table S4).

\section{Factors Contributing to Differences Over Time Within Genera}

The ordination analysis of the comb microbiota results showed that communities were particularly different across years, which was consistent across the three termite genera (Figs. 2a and S4). However, several comb communities from geographically distant sites ( $>450 \mathrm{~km}$ apart) were remarkably similar within a year (e.g. M. natalensis 138 and M. natalensis 134 and Odontotermes sp. 128 and Odontotermes sp. 130;
Figs. 2a and S4). Given that the majority of bacterial sequences identified within combs were shared with gut communities, we expected that comb communities would strongly mirror gut communities. The latter, however, were consistent in composition over time.

We were not able to quantitatively explore community similarities between combs and guts in PCoA space due to the use of different sequencing techniques (Fig. 2), but additional PCoA analyses suggested that both changes to the relative abundances of bacterial families shared with gut communities (Fig. 4a) and the sets of bacteria unique to combs (Fig. 4c) contribute to the separation of fungus comb microbiotas from the same termite species across the 2 years of sampling. The analyses did, however, suggest that the set of shared bacteria might have a larger impact on the temporal variation (Fig. 4a).

The factors contributing to community changes over time in a manner that leads to consistent communities within termite species within years are yet to be fully addressed. Nevertheless, changes in the composition of plant material ingested by the termites may affect what bacteria are deposited in the combs. If habitats offer different plant material as termite forage, this could also contribute to differences between years and across different sites, depending on whether such changes influence comb communities. Changes in the surrounding soil and mound microbiotas may also influence comb communities when combs come in contact with the soil, and these changes could be driven by environmental conditions, plant cover and composition, and macrofauna [37]. Differences in temperature, rainfall, or humidity may also affect soil microbiotas surrounding termite mounds. Data from the South African Weather Service (not shown) indicate that there were no discernible differences in maximum or minimum temperatures, daily rainfall, or humidity in the 12 months prior to the 2011 and 2013 collections. This suggests that climatic differences may not be the main reason for differences in comb community composition in our study; however, we cannot exclude that local conditions may affect the properties and growth conditions for bacteria communities within combs.

\section{Conclusions}

Fungus comb microbiotas are generally very similar within termite genera and even species within years, but this pattern is disrupted over time by factors that our current knowledge of communities and environmental conditions cannot address. In contrast, and as expected, gut microbiotas were persistent over time and shaped by termite host species. Large proportions of the gut bacterial communities are shared with fungus combs, presumably transferred during fungus comb formation by termite deposits of primary faeces. Despite these gut community 
deposits to fungus comb communities, the latter harboured relatively small proportions of bacteria families present in termite guts, probably because fungus combs are more exposed and potentially receive a continuous influx of bacteria from the surroundings. The possible functions of fungus comb bacterial communities remain unknown, and future analyses of their metabolic activity will hopefully allow establishment of possible symbiotic roles.

Acknowledgments We thank Z. Wilhelm de Beer, Michael J. Wingfield, and the staff and students at the Forestry and Agricultural Biotechnology Institute, University of Pretoria, for hosting field work, Sze Huei Yek, Zander Human, Thijs Gruntjes, and Jens Ringelberg for help with excavations, the Oerlemans family (Mookgophong) for permission to sample colonies at their farm, the South African Weather Service for the meteorological records, Judith S. Opp at the University of Michigan Medical School for laboratory assistance in the MiSeq library preparation, Sylvia Mathiasen, Karin Vestberg, and Panagiotis Sapountzis for laboratory assistance, and Rachelle Adams, Panagiotis Sapountzis, and Jacobus J. Boomsma for comments on a previous draft of the manuscript. This work was supported by a $\mathrm{PhD}$ stipend from the Department of Biology, University of Copenhagen, and the Danish National Research Foundation (DNRF57) to SO, and a STENO grant from The Danish Council for Independent Research | Natural Sciences, and a Villum Kann Rasmussen Young Investigator Programme grant to MP.

Authors' Contributions SO designed the study, performed DNA extractions, prepared libraries, analysed the data, prepared the figures, contributed to the interpretation of the sequencing results, and wrote the first draft of the manuscript. LHH and SJS contributed primers, tags, adaptors, and reagents for 454 pyrosequencing and assisted with library preparation. MP designed the study, funded the experimental work, and contributed to the interpretation of the results, the preparation of figures, and to writing the manuscript.

Open Access This article is distributed under the terms of the Creative Commons Attribution 4.0 International License (http:// creativecommons.org/licenses/by/4.0/), which permits unrestricted use, distribution, and reproduction in any medium, provided you give appropriate credit to the original author(s) and the source, provide a link to the Creative Commons license, and indicate if changes were made.

\section{References}

1. Batra LR, Batra SWT (1966) Fungus-growing termites of tropical India and associated fungi. J Kansas Entomol Soc 39(4):725-738. doi: $10.2307 / 25083581$

2. Wood TG, Sands WA (1978) The role of termites in ecosystems. In: Brian MV (ed) Production ecology of ants and termites. Cambridge University Press, Cambridge, pp 245-292

3. Abe T, Matsumoto T (1979) Studies on the distribution and ecological role of termites in a lowland rain forest of west Malaysia. 3. Distribution and abundance of termites in Pasoh Forest Reserve. Jpn J Ecol 29(3):337-351. doi:10.1007/BF00345187

4. Buxton RD (1981) Termites and the turnover of dead wood in an arid tropical environment. Oecologia 51(3):379-384. doi:10.1007/ BF00540909

5. Nobre T, Rouland-Lefèvre C, Aanen DK (2011) Comparative biology of fungus cultivation in termites and ants. In: Bignell D, Roisin
Y, Lo N (eds) Biology of termites: a modern synthesis, 2nd edn. Springer, Dordrecht, pp 193-210

6. Brune A, Ohkuma M (2011) Role of the termite gut microbiota in symbiotic digestion. In: Bignell D, Roisin Y, Lo N (eds) Biology of termites: a modern synthesis, 2nd edn. Springer, Dordrecht, pp 439-475

7. Hongoh Y (2011) Toward the functional analysis of uncultivable, symbiotic microorganisms in the termite gut. Cell and Mol Life Sci 68(8):1311-1325. doi:10.1007/s00018-011-0648-Z

8. Liu N, Zhang L, Zhou H, Zhang M, Yan X, Wang Q, Long Y, Xie L, Wang S, Huang Y, Zhou Z (2013) Metagenomic insights into metabolic capacities of the gut microbiota in a fungus-cultivating termite Odontotermes yunnanensis. PLoS ONE 8(7), e69184. doi:10. 1371/journal.pone.0069184

9. Darlington J (1994) Nutrition and evolution in fungus-growing termites. In: Hunt JH, Nalepa CA (eds) Nourishment and evolution in insect societies. Westview Press, Boulder, CO, USA, pp 105-130

10. Badertscher S, Gerber C, Leuthold RH (1983) Polyethism in food supply and processing in termite colonies of Macrotermes subhyalinus (Isoptera). Behav Ecol Sociobiol 12(2):115-119. doi: 10.1007/BF00343201

11. Sands WA (1960) The initiation of fungus comb construction in laboratory colonies of Ancistrotermes guineensis (Silvestri). Insectes Soc 7:251-263. doi:10.1007/BF02224496

12. Taprab Y, Johjima T, Maeda Y, Moriya S, Trakulnaleamsai S, Noparatnaraporn N, Ohkuma M, Kudo T (2005) Symbiotic fungi produce laccases potentially involved in phenol degradation in fungus combs of fungus-growing termites in Thailand. Appl Environ Microbiol 71(12):7696-7704. doi:10.1128/aem.71.12.7696-7704.2005

13. Rouland-Lefèvre C, Inoue T, Johjima T (2006) Termitomyces/termite interactions. In: König H, Varma A (eds) Intestinal microorganisms of soil invertebrates, vol 6. Springer-Verlag, Berlin Heidelberg, pp 335-350

14. Leuthold RH, Badertscher S, Imboden H (1989) The inoculation of newly formed fungus comb with Termitomyces in Macrotermes colonies (Isoptera, Macrotermitinae). Insectes Soc 36:328-338

15. Rouland-Lefèvre C, Bignell DE (2001) Cultivation of symbiotic fungi by termites of the subfamily Macrotermitinae. In: Seckbach J (ed) Symbiosis. Kluwer, Netherlands, pp 731-756

16. Dietrich C, Köhler T, Brune A (2014) The cockroach origin of the termite gut microbiota: patterns in bacterial community structure reflect major evolutionary events. Appl Environ Microbiol. doi: 10.1128/aem.04206-13

17. Otani S, Mikaelyan A, Nobre T, Hansen L, Kone N, Sørensen S, Aanen DK, Boomsma JJ, Brune A, Poulsen M (2014) Identifying the core microbial community in the gut of fungus-growing termites. Mol Ecol 23(18):4631-4644

18. Matsumoto $\mathrm{T}$ (1976) The role of termites in an equatorial rain forest ecosystem of West Malaysia. I. Population density, biomass, carbon, nitrogen and calorific content and respiration rate. Oecologia 22(2):153-178. doi:10.2307/4215207

19. Eggleton P, Bignell DE, Sands WA, Mawdsley NA, Lawton JH, Wood TG, Bignell NC (1996) The diversity, abundance and biomass of termites under differing levels of disturbance in the Mbalmayo Forest Reserve, Southern Cameroon. Philos Trans R Soc Lond B Biol Sci 351(1335):51-68. doi:10.1098/rstb.1996.0004

20. Donovan SE, Eggleton P, Bignell DE (2001) Gut content analysis and a new feeding group classification of termites. Ecol Entomol 26(4):356-366. doi:10.1046/j.1365-2311.2001.00342.x

21. Hyodo F, Tayasu I, Inoue T, Azuma JI, Kudo T, Abe T (2003) Differential role of symbiotic fungi in lignin degradation and food provision for fungus-growing termites (Macrotermitinae: Isoptera). Funct Ecol 17(2):186-193. doi:10.1046/j.1365-2435.2003.00718.x

22. Hongoh Y (2010) Diversity and genomes of uncultured microbial symbionts in the termite gut. Biosci Biotechnol Biochem 74(6): $1145-1151$ 
23. Korb J (2011) Termite mound architecture, from function to construction. In: Bignell D, Roisin Y, Lo N (eds) Biology of termites: a modern synthesis. Springer, Dordrecht, pp 349-373

24. Li H, Dietrich C, Zhu N, Mikaelyan A, Ma B, Pi R, Liu Y, Yang M, Brune A, Mo J (2015) Age polyethism drives community structure of the bacterial gut microbiota in the fungus-cultivating termite Odontotermes formosanus. Environ Microbiol: n/a-n/a. doi:10. 1111/1462-2920.13046

25. Ruelle JE, Coaton WGH, Sheasby JL (1975) National survey of the Isoptera of southern Africa. 8. The genus Macrotermes Holmgren (Termitida: Macrotermitinae). Cimbebasia 3:73-94

26. Hausberger B, Kimpel D, van Neer A, Korb J (2011) Uncovering cryptic species diversity of a termite community in a West African savanna. Mol Phylogenet Evol 61(3):964-969. doi:10.1016/j. ympev.2011.08.015

27. Liu H, Beckenbach AT (1992) Evolution of the mitochondrial cytochrome oxidase II gene among 10 orders of insects. Mol Phylogenet Evol 1(1):41-52

28. Simon C, Frati F, Beckenbach A, Crespi B, Liu H, Flook P (1994) Evolution, weighting, and phylogenetic utility of mitochondrial gene sequences and a compilation of conserved polymerase chain reaction primers. Ann Entomol Soc Am 87(6):651-701

29. Edgar RC (2004) MUSCLE: multiple sequence alignment with high accuracy and high throughput. Nucleic Acids Res 32(5): 1792-1797. doi:10.1093/nar/gkh340

30. Page RDM (1996) Tree View: an application to display phylogenetic trees on personal computers. Comput Appl Biosci 12(4):357358. doi:10.1093/bioinformatics/12.4.357

31. Hansen CH, Krych L, Nielsen DS, Vogensen FK, Hansen LH, Sorensen SJ, Buschard K, Hansen AK (2012) Early life treatment with vancomycin propagates Akkermansia muciniphila and reduces diabetes incidence in the NOD mouse. Diabetologia 55(8):22852294. doi:10.1007/s00125-012-2564-7

32. Schloss PD, Westcott SL, Ryabin T, Hall JR, Hartmann M, Hollister EB, Lesniewski RA, Oakley BB, Parks DH, Robinson CJ, Sahl JW, Stres B, Thallinger GG, Van Horn DJ, Weber CF (2009) Introducing mothur: open-source, platform-independent, community-supported software for describing and comparing microbial communities. Appl Environ Microbiol 75(23):7537-7541. doi:10.1128/aem.01541-09

33. Mikaelyan A, Köhler T, Lampert N, Rohland J, Boga H, Meuser K, Brune A Classifying the bacterial gut microbiota of termites and cockroaches: a curated phylogenetic reference database $($ Dict $D b)$. Syst Appl Microbiol. doi: http://dx.doi.org/10.1016/j.syapm.2015.07.004

34. Hongoh Y, Ekpornprasit L, Inoue T, Moriya S, Trakulnaleamsai S, Ohkuma M, Noparatnaraporn N, Kudo T (2006) Intracolony variation of bacterial gut microbiota among castes and ages in the fungus-growing termite Macrotermes gilvus. Mol Ecol 15(2):505516. doi:10.1111/j.1365-294X.2005.02795.x

35. Miyata R, Noda N, Tamaki H, Kinjyo K, Aoyagi H, Uchiyama H, Tanaka H (2007) Influence of feed components on symbiotic bacterial community structure in the gut of the wood-feeding higher termite Nasutitermes takasagoensis. Biosci Biotechnol Biochem 71(5):1244-1251

36. Huang XF, Bakker MG, Judd TM, Reardon KF, Vivanco JM (2013) Variations in diversity and richness of gut bacterial communities of termites (Reticulitermes flavipes) fed with grassy and woody plant substrates. Microb Ecol 65(3):531-536. doi:10.1007/s00248-0130219-y
37. Lavelle P, Spain A (2001) Soil ecology. Kluwer Academic Publishers, Dordrecht, The Netherlands

38. Aanen DK, Eggleton P (2005) Fungus-growing termites originated in African rain forest. Curr Biol 15(9):851-855

39. Visser AA, Nobre T, Currie CR, Aanen DK, Poulsen M (2012) Exploring the potential for Actinobacteria as defensive symbionts in fungus-growing termites. Microbiol Ecol 63(4):975-985. doi:10. 1007/s00248-011-9987-4

40. R Core Team (2013). R: A language and environment for statistical computing. R Foundation for Statistical Computing, Vienna, Austria. ISBN 3-900051-07-0, URL http://www.R-project.org/.

41. Kozich JJ, Westcott SL, Baxter NT, Highlander SK, Schloss PD (2013) Development of a dual-index sequencing strategy and curation pipeline for analyzing amplicon sequence data on the MiSeq Illumina sequencing platform. Appl Environ Microbiol 79(17):5112-5120

42. Koenigsknecht MJ, Theriot CM, Bergin IL, Schumacher CA, Schloss PD, Young VB (2015) Dynamics and establishment of Clostridium difficile infection in the murine gastrointestinal tract. Infect Immun 83(3):934-941

43. Poulsen M, Hu H, Li C, Chen Z, Xu L, Otani S, Nygaard S, Nobre T, Klaubauf S, Schindler PM, Hauser F, Pan H, Yang Z, Sonnenberg ASM, de Beer ZW, Zhang Y, Wingfield MJ, Grimmelikhuijzen CJP, de Vries RP, Korb J, Aanen DK, Wang J, Boomsma JJ, Zhang G (2014) Complementary symbiont contributions to plant decomposition in a fungus-farming termite. Proc Natl Acad Sci 111(40):14500-14505

44. Aanen DK, Eggleton P, Rouland-Lefèvre C, Guldberg-Frøslev T, Rosendahl S, Boomsma JJ (2002) The evolution of fungus-growing termites and their mutualistic fungal symbionts. Proc Natl Acad Sci 99(23): 14887-14892

45. Iqbal N, Saeed S, Evans TA, Kwon YJ (2015) Foraging activity and population estimation of Microtermes mycophagus Desneux (Isoptera: Termitidae: Macrotermitinae) in Multan, Punjab, Pakistan. Entomol Res 45(2):51-57

46. Shinzato N, Muramatsu M, Matsui T, Watanabe Y (2007) Phylogenetic analysis of the gut bacterial microflora of the fungus-growing termite Odontotermes formosanus. Biosci Biotechnol Biochem 71(4):906-915

47. Makonde HM, Boga HI, Osiemo Z, Mwirichia R, Mackenzie LM, Goker M, Klenk HP (2013) 16S-rRNA-based analysis of bacterial diversity in the gut of fungus-cultivating termites (Microtermes and Odontotermes species). Antonie Van Leeuwenhoek 104(5):869883. doi:10.1007/s10482-10013-10001-10487

48. Brune A (2014) Symbiotic digestion of lignocellulose in termite guts. Nat Rev Micro 12(3):168-180

49. Mitchell BL (1980) Report on a survey of the termites of Zimbabwe. Occasional Papers of the National Museums and Monuments of Rhodesia (Natural Sciences) 6(5):187-323

50. Uys V (2002) A guide to the termite genera of Southern Africa. Plant protection research institute handbook. No. 15. Agricultural Research Council, Pretoria

51. Mikaelyan A, Dietrich C, Köhler T, Poulsen M, Sillam-Dussès D, Brune A (2015) Diet is the primary determinant of bacterial community structure in the guts of higher termites. Mol Ecol: n/a-n/a. doi:10.1111/mec.13376

52. Makonde H, Mwirichia R, Osiemo Z, Boga H, Klenk H-P (2015) 454 Pyrosequencing-based assessment of bacterial diversity and community structure in termite guts, mounds and surrounding soils. SpringerPlus 4:471 